\title{
Substance of Abuse
}

National Cancer Institute

\section{Source}

National Cancer Institute. Substance of Abuse. NCI Thesaurus. Code C1816.

Any substance, including any illegal, prescription, over-the-counter agent or any other compound, used for the purpose other than indicated or used in quantities other than directed. Substances from various drug classes can be abused, such as opioids, amphetamines, analgesics, benzodiazepines, barbiturates, hallucinogens, steroids, tobacco products and alcoholic substances. A substance of abuse may act on the central nervous system (CNS) or can have an effect in other parts of the body and can lead to physical and/or physiological dependence. 